\title{
A composite semiresorbable armoured scaffold stabilizes pulmonary autograft after the Ross operation: Mr Ross's dream fulfilled
}

\author{
Francesco Nappi, MD, ${ }^{\text {a }}$ Cristiano Spadaccio, MD, PhD,${ }^{\mathrm{b}}$ Massimiliano Fraldi, $\mathrm{PhD},{ }^{\mathrm{c}}$ \\ Stefania Montagnani, MD, ${ }^{\mathrm{d}}$ Pierre Fouret, MD,${ }^{\mathrm{e}}$ Juan Carlos Chachques, MD, PhD, ${ }^{\mathrm{f}}$ and \\ Christophe Acar, $\mathrm{MD}, \mathrm{PhD}^{\mathrm{g}}$
}

\begin{abstract}
Objectives: Use of resorbable external reinforcement of the pulmonary autograft during the Ross operation has been suggested, but the differential regional potential for dilation of the aorta, mainly regarding the neo-root and the neo-Valsalva sinuses, represents an unresolved issue. Auxetic materials could be useful in preventing dilation given their favorable mechanical properties. We designed a composite semiresorbable armoured bioprosthesis constituted by polydioxanone and expanded polytetrafluoroethylene and evaluated its effectiveness as a pulmonary autograft reinforcement device in an animal model of the Ross procedure.
\end{abstract}

Methods: An experimental model of the Ross procedure was performed in 20 three-month-old growing lambs. The pulmonary autograft was alternatively nonreinforced (control group $\mathrm{n}=10$ ) or reinforced with composite bioprosthesis (reinforced group $n=10$ ). Animals were followed up during growth for 6 months by angiography and echocardiography. Specific stainings for extracellular matrix and immunohistochemistry for metalloproteinase- 9 were performed.

Results: Reference aortic diameter increased from $14 \pm 1 \mathrm{~mm}$ to $19 \pm 2 \mathrm{~mm}$ over 6 months of growth. In the control group, pulmonary autograft distension (28 $\pm 2 \mathrm{~mm})$ was immediately noted, followed by aneurysm development at 6 months ( $40 \pm 2 \mathrm{~mm}, P<.001$ vs reference). In the reinforced group, an initial dilation to $18 \pm 1 \mathrm{~mm}$ was detected and the final diameter was $27 \pm 2 \mathrm{~mm}(42 \%$ increase). Two deaths due to pulmonary autograft rupture occurred in the control group. On histology, the control group showed medial disruption with connective fibrous replacement, whereas in the reinforced group compensatory intimal hyperplasia was present in the absence of intimal tears. The bioprosthesis promoted a positive matrix rearrangement process favoring neoarterialization and elastic remodeling as demonstrated on specific staining for elastin collagen and metalloproteinase-9.

Conclusions: The device adapted and functionally compensated for the characteristics of autograft growth, guaranteeing a reasonable size of the autograft at 6 months, but more important, because the device is biocompatible, it did not disrupt the biological process of growth or cause inflammatory damage to the wall. (J Thorac Cardiovasc Surg 2016;151:155-64)

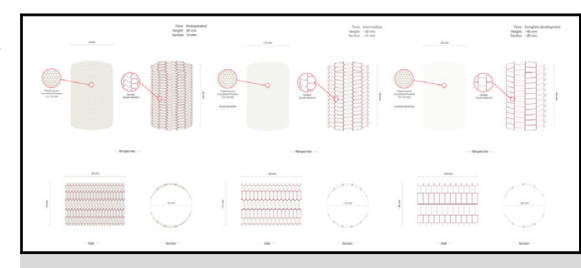

Newly developed composite semiresorbable armoured scaffold for autograft reinforcement.

Central Message

A composite semiresorbable reinforcemen prevented autograft dilation, promoting neoarterialization and respecting its growth over time.

\section{Perspective}

Reinforcement strategies currently adopted in the Ross operation are unable to match the needs of a growing structure, and they compromise the biomechanical features of the aorta. A newly designed semiresorbable PA reinforcement prevented PA dilation over time while adapting and functionally compensating for the characteristics of somatic growth and might have a potential in pediatric surgery.

See Editorial Commentary page 165.

\footnotetext{
From the ${ }^{\mathrm{a}}$ Cardiac Surgery Centre Cardiologique du Nord de Saint-Denis, Paris, France; ${ }^{b}$ Department of Cardiothoracic Surgery, Golden Jubilee National Hospital, Clydebank, Glasgow, United Kingdom; ${ }^{\mathrm{c}}$ Department of Structures and Interdisciplinary Research Center for Biomaterials, University of Napoli Federico II, Naples, Italy; 'Department of Public Health, University of Naples "Federico II," Naples, Italy; ' Department of Pathology, Hôpital de la Salpétrière, Paris, France; ' Laboratory of Biosurgical Research, Carpentier Foundation, Pompidou Hospital, University Paris Descartes, Paris, France; and ${ }^{\mathrm{g}}$ Department of Cardiovascular Surgery, Hôpital de la Salpétrière, Paris, France.
}

M.F. substantially contributed to the development of the mathematic model describing the biomechanical behavior of the composite device constituted by both resorbable and auxetic materials.

Received for publication Feb 22, 2015; revisions received Aug 10, 2015; accepted for publication Sept 12, 2015; available ahead of print Oct 23, 2015.

Address for reprints: Francesco Nappi, MD, Cardiac Surgery Centre Cardiologique du Nord de Saint-Denis, 36 Rue des Moulins Gémeaux, 93200 Saint-Denis, France (E-mail: francesconappi2@gmail.com).

$0022-5223 / \$ 36.00$

Copyright (c) 2016 by The American Association for Thoracic Surgery

http://dx.doi.org/10.1016/j.jtcvs.2015.09.084 


\section{Abbreviations and Acronyms}

ePTFE $=$ expanded polytetrafluoroethylene

PA $=$ pulmonary autograft

PDS = polydioxanone

Supplemental material is available online.

Despite the renewed attention to the Ross procedure expressed in the literature and the recent reports announcing its renaissance, the risk for pulmonary autograft (PA) dilation is still perceived as a daunting issue to be fully addressed. ${ }^{1-5}$ This complication jeopardizes the benefits related to the ability of the living PA to accompany the somatic growth of aortic structures in children and young adults, ${ }^{6}$ and to the avoidance of lifelong anticoagulation. ${ }^{7}$ Reinforcement strategies proposed to date are based on the use of synthetic materials, such as Dacron, ${ }^{8-11}$ which fails to match the biological requirements of a growing structure and dramatically compromises the native elastomechanical features of the ascending aorta. ${ }^{12} \mathrm{We}$ recently introduced the idea of using resorbable scaffolds to reinforce the $\mathrm{PA}^{13}$ in a previously validated model of the Ross operation in the growing lamb. ${ }^{14}$ Over time, polydioxanone (PDS) contained the dilation process of PA and elicited a phenomenon of histoarchitectural rearrangement of the PA, resulting in medial thickening and an increase in the elastic wall component leading to the formation of a "neovessel" with characteristics similar to the native aorta. ${ }^{14}$ However, observations by Horer and colleagues ${ }^{15}$ concerning the differential potential of dilation at the various segments of the aorta, that is, the annulus, Valsalva sinuses $(0.5 \mathrm{~mm} / \mathrm{y})$, and sinotubular junction (increase of $0.7-0.9 \mathrm{~mm} / \mathrm{y}),{ }^{15}$ outlined the need to tailor mechanical features of PA reinforcements in their different parts. This induced Horer and colleagues to reconsider their design with the aim to better adapt to the normal physiology of the aortic root, improving the resistance of the zones, which are meant to suffer from dilatative degeneration. In the context of the Ross procedure, the potential of dilation of the Valsalva sinuses is a pivotal problem because it might affect the status of the reimplanted coronaries. In addition, mechanical stress associated with progressive overstrain of the graft under systemic pressure might affect the PA integrity and the endothelialization process ${ }^{16}$; therefore, in this context, prevention of graft stretching is crucial. On the basis of a mathematic model founded on Hooke's law and Laplace equilibrium, we designed a novel reinforcement based on the combination of resorbable and auxetic synthetic materials with the aim to implement the first step of our experimentation and to overcome these unresolved issues.

Auxetic materials, such as expanded polytetrafluoroethylene (ePTFE) (Gore-Tex, WL Gore \& Associates, Newark, $\mathrm{Del}$ ), are characterized by a negative Poisson's ratio; that is, when they are stretched, they become thicker perpendicular to the applied load. This counterintuitive behavior can be observed at different levels-from single molecules to macroscopic matter-and generally can be traced to a particular internal material micro-structure, often made of interconnected thin elements arranged to form reentrant hexagonal patterns that confer to the overall material response mechanical properties, such as high deformability, high-energy absorption, and somewhat high fracture resistance.

In our settings, the auxetic material (and behavior) was obtained by means of a structural weave characterized by a negative Poisson's ratio, made of ePTFE (Gore-Tex). This structure was combined with a PDS one to create a prosthetic system able to accommodate mechanical loads due to systemic pressure, guaranteeing autograft integrity and controlling the progressive graft dilation. ${ }^{17,18}$ The rationale of this approach relies in the possibility of maintaining and enhancing the positive biological effects on vessel wall remodeling, previously shown with resorbable materials, providing additional stabilization of the neoaortic root through the use of a tailored ePTFE mesh, which allows regional somatic growth and prevents dilatative degeneration. This study was undertaken to determine the suitability and effectiveness of this newly semiresorbable PA reinforcement device in an animal model of the Ross procedure.

\section{MATERIALS AND METHODS Device}

The device consisted of an internal resorbable scaffold made with PDS and an external nonresorbable layer of ePTFE specifically designed to absorb mechanical stress and accompany graft growth. The materials used are commercially available, and they were assembled on the basis of their biophysical and elastomechanical properties. The PDS layer was arranged in a frame of hexagonal cells reinforced by an external armour of ePTFE. The layer of ePTFE was coupled externally to the bioabsorbable material and comprises a plurality of longitudinal lateral strips, having an auxetic behavior, and a plurality of transverse wires, which connect each longitudinal strip with the 2 adjacent. The layer of auxetic material was realized as a deformable matrix and applied to the bioresorbable to orient in the same direction the polymer chains and thus make the overall structure more compact. The layer of bioabsorbable material was designed to minimize radial tension and was constructed to embrace the root of the aorta, allowing its multidirectional growth.

\footnotetext{
Animal Model

Transposition of the pulmonary trunk in the aortic position was performed in 20 growing lambs as previously described. For this study, animals 8 to 10 weeks old with an average weight of $21 \pm 3 \mathrm{~kg}$ were used. ${ }^{14}$ Morphometric and cardiovascular parameters were comparable preoperatively among animals. There was no difference in hemoglobin
} 


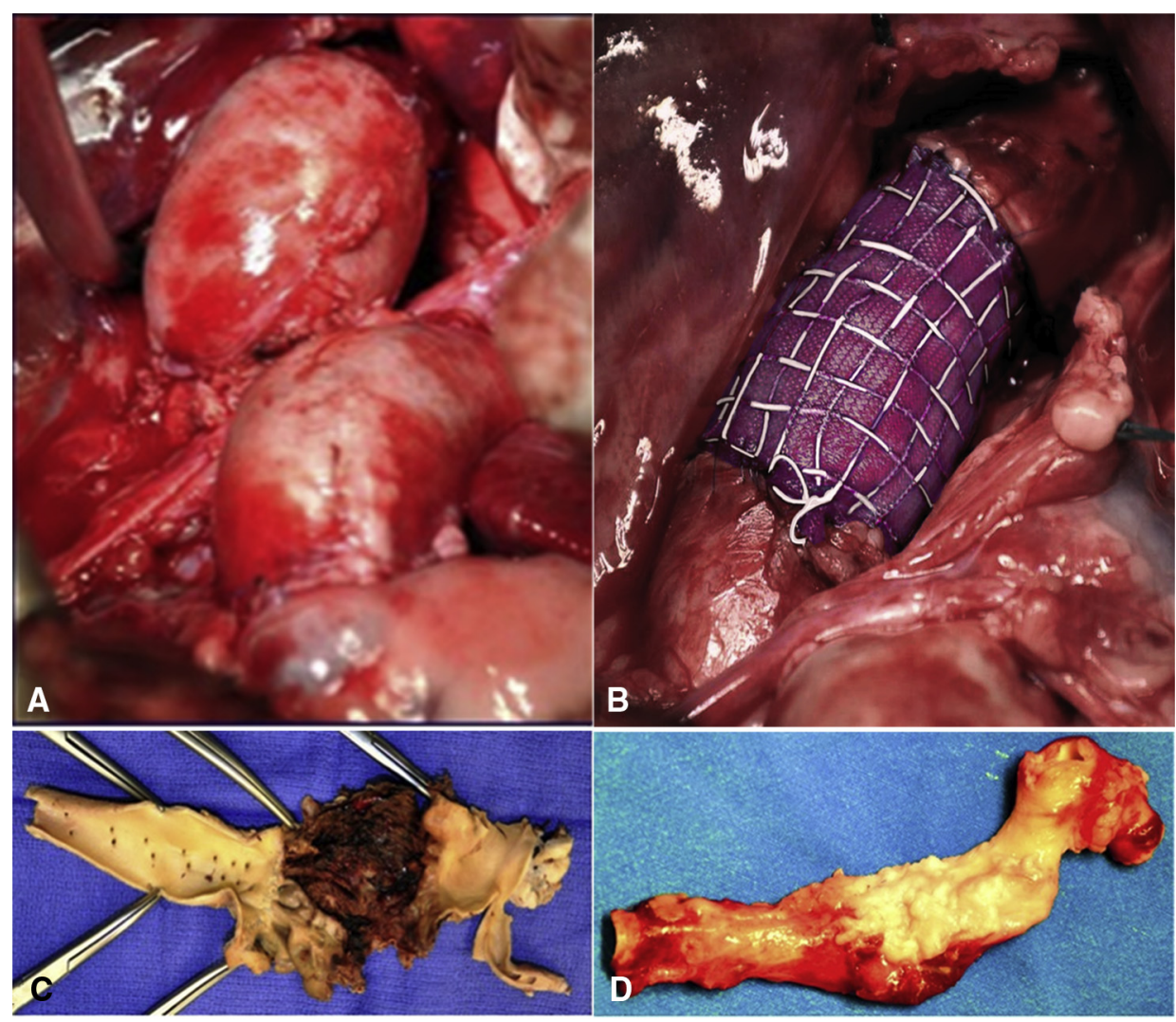

FIGURE 1. Top: Intraoperative photographs of PA implanted with no reinforcement (A) or with composite bioresorbable reinforcement (B). Bottom: Macroscopic findings after explantation at 6 months of nonreinforced PA (C) and reinforced PA (D). Note rupture and intraluminal thrombus in the nonreinforced group $(\mathrm{C})$.

levels and ventricular function. Briefly, anesthesia was induced with sodium thiopental $(6-8 \mathrm{mg} / \mathrm{kg})$ via the internal jugular maintained with isoflurane $(1 \%$ to $2.5 \%)$ inhalation. Animals were intubated and received $100 \mathrm{mg}$ of lidocaine as prophylaxis against rhythm disturbance. The heart was approached via a left thoracotomy, and cardiopulmonary bypass was established between the descending aorta and the right atrium. The cerebral circulation of the animal was maintained on a beating heart. The pulmonary trunk was harvested and transposed into the descending aorta with an endto-end anastomosis in 5-0 Prolene. A fresh pulmonary artery homograft, explanted from animals killed on the same day for another experimental study, was inserted to reconstruct the right outflow tract, with a proximal and distal end-to-end anastomosis in 5-0 Prolene, as in the Ross operation. Ten lambs received the prosthetic reinforcement of the PA. The mesh was cut into a rectangle measuring $20 \mathrm{~mm}$ in height corresponding to the height of the PA, rolled on a metallic candle, and then fixed with a suture to create a cylinder with an internal diameter of $10 \mathrm{~mm}(20 \mathrm{~mm}$ height and $10 \mathrm{~mm}$ diameter). The PA was then inserted into the fibrillar cylinder and anastomosed, suturing both its margins and those of the prosthetic structure to the PA trunk (Figures 1 and 2). Ten additional animals did not receive any reinforcement and served as the control nonreinforced group. All animal experiments were performed following the guidelines for animal care and handling, and the protocol was approved by the institutional animal care committee.

\section{Data Acquisition}

Animals were assessed with angiography and echocardiography at 2 time points, at day 0 and 6 months postoperatively, respectively. Selection of this time frame has been guided by 2 main points. First, 6 months is the approximate time required for lambs to reach a weight of $60 \mathrm{~kg}$, which might reliably reflect the body mass of the average human patient normally undergoing the Ross operation. Second, considering the aim to investigate the effect of a resorbable reinforcement and the time for resorption reported for PDS, we selected an end point of 6 months because this should have allowed enough time to complete the resorption process. This would have permitted us to analyze histologically the samples after a total resorption of the meshes applied. Three averaged echocardiographic and angiographic measurements were taken by 2 independent observers blinded in regard to the treatment group. An inter-rater reliability analysis using the Kappa statistic was performed to determine consistency among raters. $\mathrm{K}$ coefficient was 0.82 , indicating adequate agreement. Eventually, animals were humanely killed and tissue was harvested for histopathologic analysis. Measurements of the ascending aorta and descending aorta proximally and distally to the autograft were made to obtain a reference to be compared with the diameter of the PA. A weight curve for each animal during the growing period was in parallel processed. Hematoxylin-eosin, Masson's trichrome, Mallory, and Picrosirius red stainings were performed after explantation. Further immunohistochemistry for metalloproteinase9 was also performed to elucidate extracellular matrix remodeling phenomena. Continuous variables were compared using the paired Student $t$ test. Analysis was performed with SPSS version 20.0 software for Mac (SPSS Inc, Chicago, Ill).

\section{RESULTS}

According to the growth curve, animal weights were doubled $(21 \pm 3 \mathrm{~kg}$ at day 0 and $45 \pm 7 \mathrm{~kg}$ at 6 months $)$ 

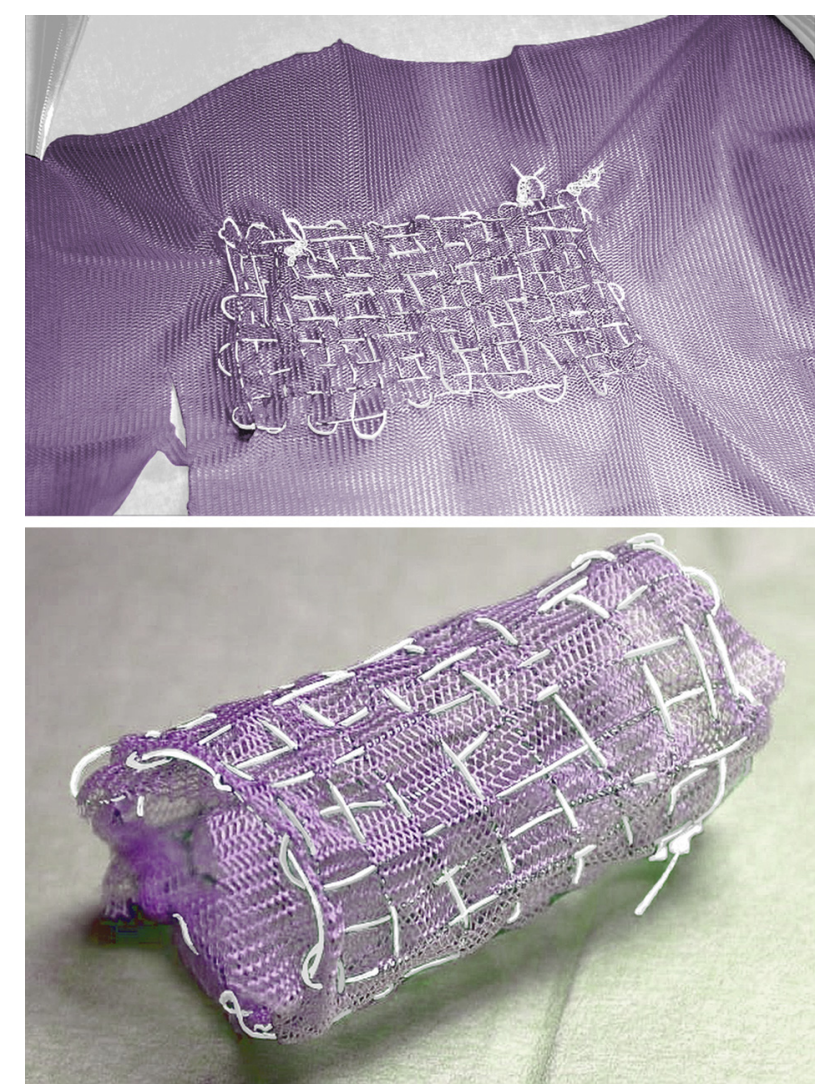

FIGURE 2. Photograph of handmade composite PDS PTFE (Gore-Tex) (WL Gore \& Associates, Newark, Del) reinforcement before implantation.

In the control group, 2 animals died of aneurysmatic rupture of the PA, whereas there was no mortality in the reinforced group. On angiographic analysis, the reference aortic diameter increased from $14 \pm 1 \mathrm{~mm}$ to $19 \pm 2 \mathrm{~mm} \mathrm{(34 \%} \mathrm{in-}$ crease). In the control group, PA distension $(28 \pm 2 \mathrm{~mm})$ was immediately noted, followed by aneurysm development at 6 months $(40 \pm 2 \mathrm{~mm}, P<.0001$ vs reference, $57 \%$ diameter increase with respect to day 0 ). In the reinforced group, an initial dilation to $18 \pm 1 \mathrm{~mm}$ was detected, and the final diameter was $27 \pm 2 \mathrm{~mm}$ (42\% increase) (Tables 1 and 2). Likewise, echocardiography showed an

TABLE 1. Comparison of echocardiographic and angiographic measurements between control and polydioxanone polytetrafluoroethylene (Gore-Tex)-reinforced groups

\begin{tabular}{|c|c|c|c|}
\hline & $\begin{array}{l}\text { Control } \\
(n=10)\end{array}$ & $\begin{array}{c}\text { PDS PTFE } \\
\text { (Gore-Tex; WL } \\
\text { Gore \& Associates, } \\
\text { Newark, Del) } \\
(\mathbf{n}=\mathbf{1 0})\end{array}$ & $P$ value \\
\hline Echography day 0 & $31 \pm 1.87$ & $17 \pm 2.66$ & $<.0001$ \\
\hline Echography month 6 & $42.3 \pm 3.18$ & $29.3 \pm 3.68$ & .0002 \\
\hline Angiography day 0 & $28.2 \pm 2$ & $18.1 \pm 0.98$ & $<.0001$ \\
\hline Angiography month 6 & $40.2 \pm 2.14$ & $27.1 \pm 1.99$ & $<.0001$ \\
\hline
\end{tabular}

PDS, Polydioxanone; PTFE, polytetrafluoroethylene.
TABLE 2. Comparison of echocardiographic and angiographic measurements at day 0 and 6 months postoperatively in both control and polydioxanone Gore-Tex-reinforced groups

\begin{tabular}{lccr}
\hline & Day 0 & Month 6 & P value \\
\hline Control & & & \\
Echography & $31 \pm 1.87$ & $42.3 \pm 3.18$ & .0003 \\
$\quad$ Angiography & $28.2 \pm 2$ & $40.2 \pm 2.14$ & $<.0001$ \\
PDS Gore-Tex & & & \\
Echography & $17 \pm 2.66$ & $29.3 \pm 3.68$ & $<.0001$ \\
Angiography & $18.1 \pm 0.98$ & $27.1 \pm 1.99$ & $<.0001$ \\
\hline$P D S$, Polydioxanone. & & &
\end{tabular}

immediate burst dilation of the control with a diameter of $31 \pm 2 \mathrm{~mm}$ and late aneurysmal degeneration of $42 \pm 3 \mathrm{~mm}$. The reinforced group maintained $17 \pm 3 \mathrm{~mm}$ immediately after implantation and $29 \pm 4 \mathrm{~mm}$ at 6 months (Tables 1 and 2, Figure 3).

On macroscopic analysis, specimens from the control group were severely dilated with intimal thinning and intraluminal thrombus (Figure 1,C). Two ruptures were detected. Conversely, reinforced PA did not show any particular abnormalities macroscopically (Figure 1,D). On histology, medial disruption with connective fibrous replacement of the muscular layers and lymphoid infiltrates was observed in the nonreinforced group (Figure 4). In the reinforced group, compensatory intimal hyperplasia was present in the absence of intimal tears, and remnants of the slowly resorbable PDS suture were detected in the adventitia (Figure 4). Of note, Masson's trichrome stain showed a rearrangement of the tunica media that displayed a dense fibrous network mainly constituted by elastin organized in concentric lamellae, suggesting an elastic remodeling phenomenon of the PA (Figure 4). Specific staining confirmed increased content of elastin with respect to control, with fibers organized in a compact layer at the level of the "elastic zone" of the vessel (Figure 5). In parallel, reinforced PA showed an overexpression of metalloproteinase-9 with respect to control, suggesting an underlying process of extracellular matrix remodeling and confirming the impression of elastic remodeling and arterialization (Figure 5). In addition, inflammatory infiltrate was scarce with rare macrophages or monocytes colonizing the reinforced arterial wall, reliably suggesting that the biomaterial did not elicit an exuberant inflammatory foreign-body reaction over time.

\section{DISCUSSION}

The idea of a semiresorbable composite scaffold originated from the need to reinforce the PA during the Ross procedure, providing increased stabilization and solidity of the neoaortic root and preventing its dilation due to systemic pressure. Previous experience with a prosthetic Dacron graft with an artificial aortic root configuration (Valsalva graft) as external reinforcement of the PA has been reported 

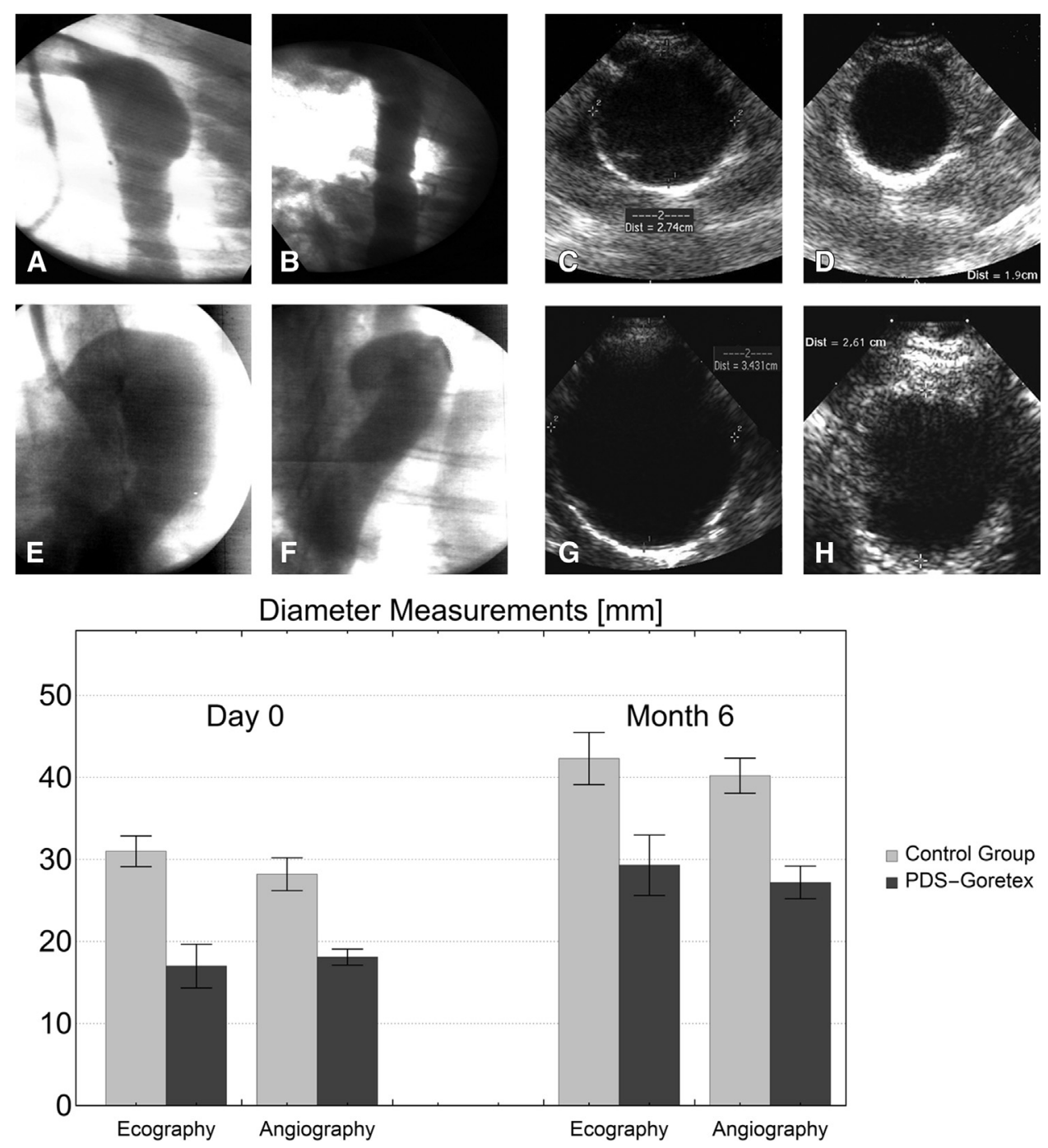

FIGURE 3. Top: A, B, E, and F, Angiographic images. A, Nonreinforced PA at day 0. E, Nonreinforced PA at month 6. B, Reinforced PA at day 0. F, Reinforced PA at month 6. Note the uniformity of the vessel profile in case of reinforcement (B, F) and loss of physiologic shape, prone to aneurysmal complication (A, E). C, D, G, and H, Echocardiographic images (vessel cross-sections). C, Nonreinforced PA at day 0. G, Nonreinforced PA at month 6. D, Reinforced PA at day 0. H, Reinforced PA at month 6. Note the severe diameter dilation in the nonreinforced case (C, G). Bottom: Bar-chart with synoptic view of the 10 data points for each group analyzed, referring to angiographic and echographic images. PDS, Polydioxanone.

by Carrel and colleagues. ${ }^{19,20}$ This approach was attempted with the aim to prevent neoaortic root dilatation and the dynamic function of Valsalva sinuses. This technique would allow the most physiologic pressure and flow patterns within the autograft with respect to a straight Dacron graft. ${ }^{9,10,21,22}$ Indeed, the autograft encased in a straight Dacron prosthetic graft was dramatically impaired in its pulsatility and compliance. Moreover, we previously demonstrated that the Dacron graft and other synthetic polyesters severely impair aortic compliance when used as vascular replacement ${ }^{16}$ and elicit a strong inflammatory reaction with significant damage to the vessel wall when used as a PA reinforcement. ${ }^{14,23}$ From these standpoints, we focused on improving the biomechanical behavior of the reinforced PA using a composite biodegradable and auxetic material.

This composite prosthesis prevented PA dilation while resorption of its PDS layer promoted a connective remodeling of the PA wall, resulting in a "neovessel" with increased elastin content and therefore potentially improved biomechanical properties. With the aim to describe the characteristic of the PA subjected to systemic pressure and to predict and tailor properties of its potential reinforcement, a mathematic model has been developed on the basis of experimental findings and preliminary research on bioresorbable reinforcement of the PA in growing animals. ${ }^{14}$ In the Ross root procedure, the PA might be considered as an extensible solid cylinder to which the Hooke's law for linear-elastic 

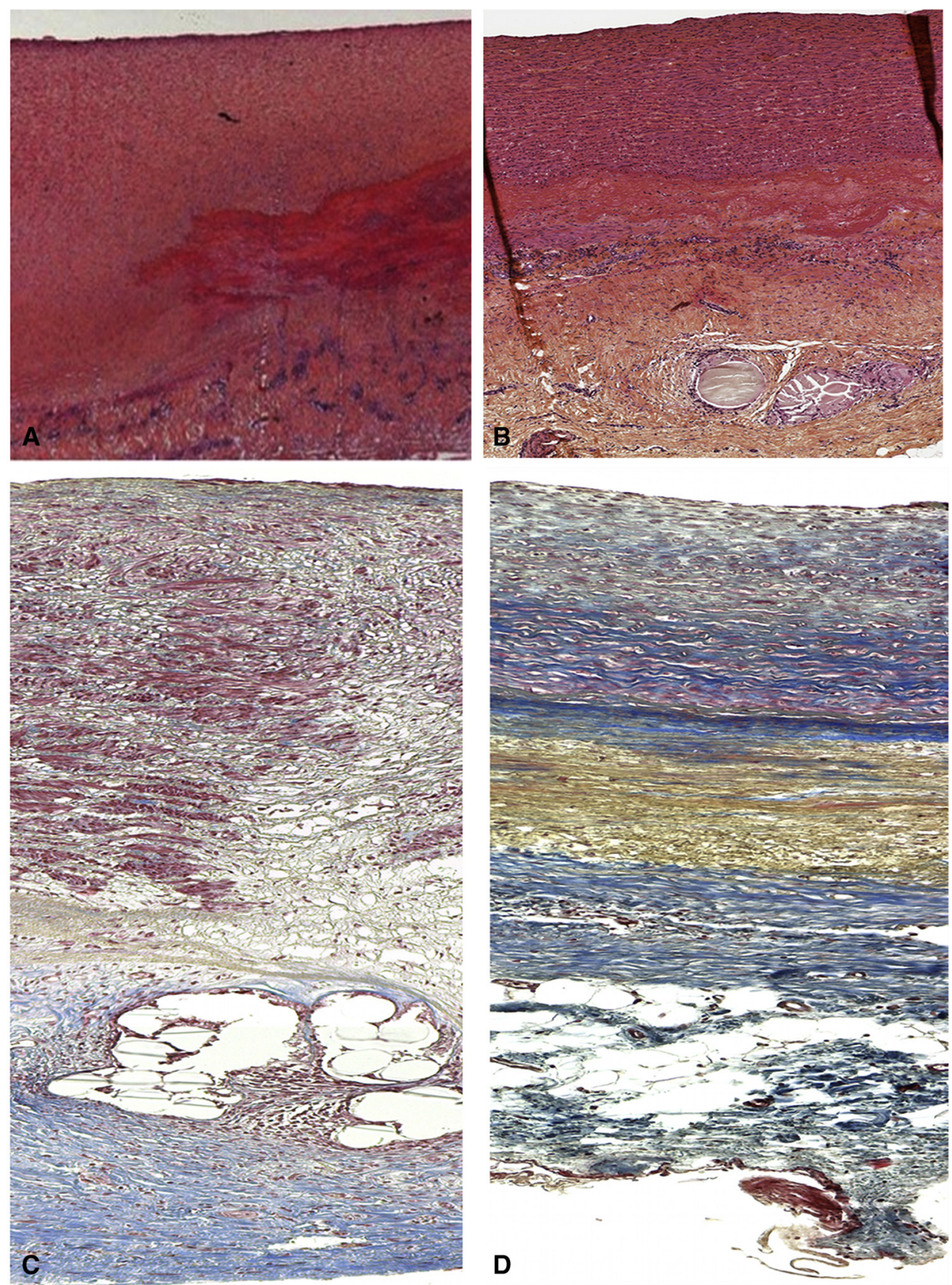

FIGURE 4. Microphotographs of hematoxylin-eosin (top) and Masson's trichrome (bottom) stainings. A, Nonreinforced control group. Note intimal fibrosis, medial disruption, and inflammatory infiltrates. B, Reinforced group. Note remnants of PDS and compensatory intimal hyperplasia. C, Nonreinforced group Trichrome staining showing connective derangement and fibrosis. D, Reinforced group showing higher connective organization with collagen (blue) dense distribution and elastin fibers (yellow) compactly distributed in the tunica media.

bodies and membranes might be applied. According to our angiographic measurements and on the basis of the histologic results, we were able to extrapolate a stress strain curve (Figure 6) representing the relationship between the stress $\vartheta$ and the deformation to which the PA is subjected. Figure 6, $A$ depicts the behavior of the nonreinforced PA, with A indicating the traits of elastic behavior, B indicating the nonelastic behavior, and $\mathrm{C}$ indicating the point of rupture. The region included under $\mathrm{ABC}$ represents the nonelastic region. The release phase (hysteresis) starts before point B. Conversely, when a crosslinked bioresorbable prosthesis realized in PDS is applied on the system, the curve modifies toward a more elastic behavior, as represented in Figure 6, $B$, with $\mathrm{A}^{\prime}, \mathrm{B}^{\prime}$, and $\mathrm{C}^{\prime}$ showing 

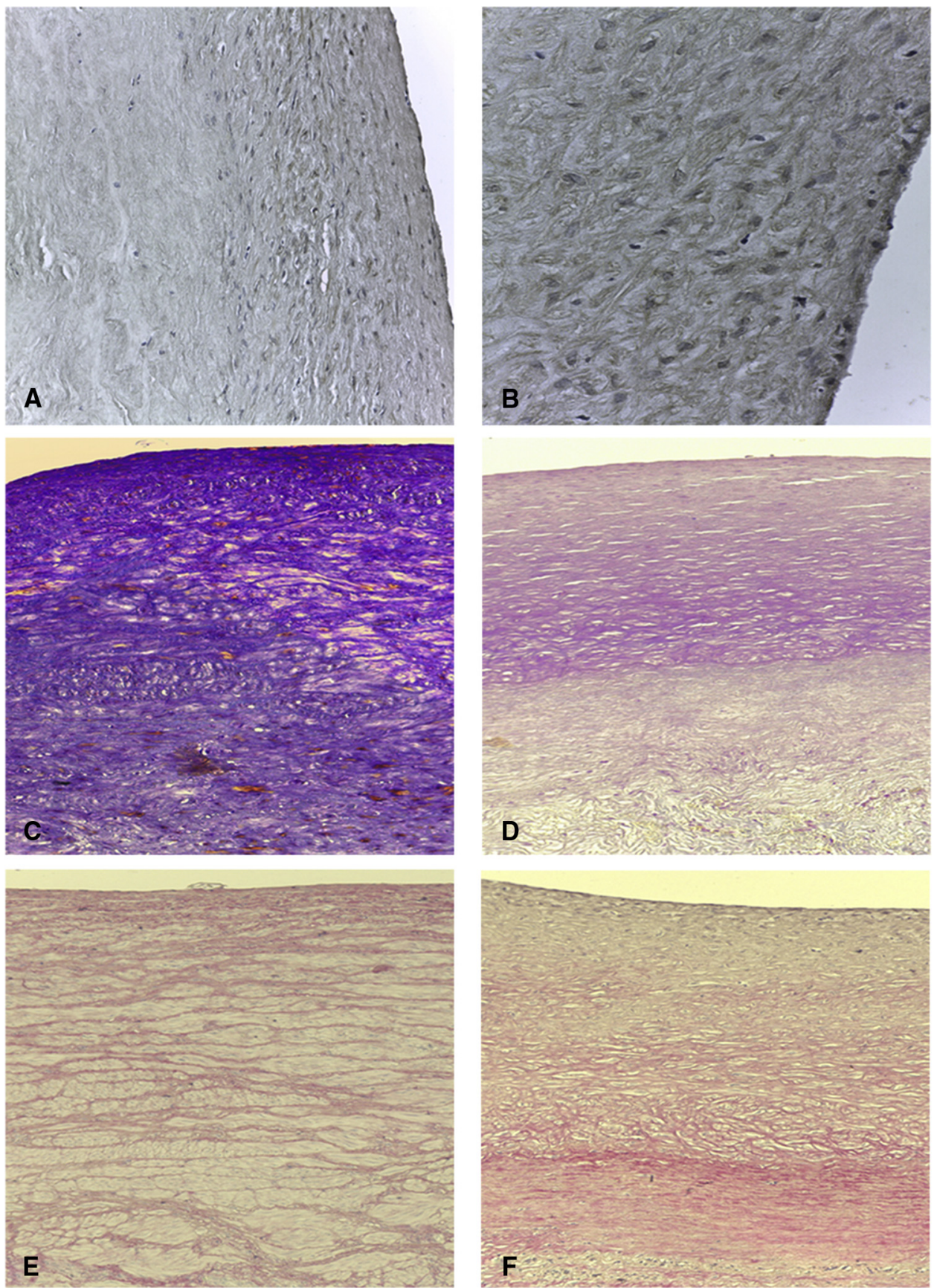

FIGURE 5. Histologic elastic remodeling. Top: Immunohistochemistry for metalloproteinase-9. A, Nonreinforced control group. B, Reinforced group. Note metalloproteinase- 9 overexpression in the reinforced group suggesting an active process of extracellular matrix remodeling. Middle: Mallory's staining. C, Nonreinforced control group showing predominant fibrosis based on collagen fibers (blue). D, Reinforced group demonstrating increased content of elastin fiber (pink). Bottom: Picrosirius red staining. E, Nonreinforced control group showing dispersed collagen fibers. F, Reinforced group compact collagen organization in the "elastic zone" of the vessel and less pronounced cellular infiltrate.

the elastic behavior, nonelastic behavior, and breakage of the bioresorbable prosthesis, respectively. Therefore, application of a bioresorbable reinforcement is able to modify the behavior of the curve of distensible materials, such as vessel wall, obtaining an increase in their elastic properties. This is observed in the curve as the shift of A toward A' (increased elasticity and compliance) and the maximum distensibility point $\mathrm{C}$ toward $\mathrm{C}^{\prime}$, as the reinforcement effectively prevented massive dilation. This provokes a reduction in the critical area determined by the fall of the curve (Figure 6,B). The mechanisms underlying this phenomenon might be found in the application of Hooke's law to 


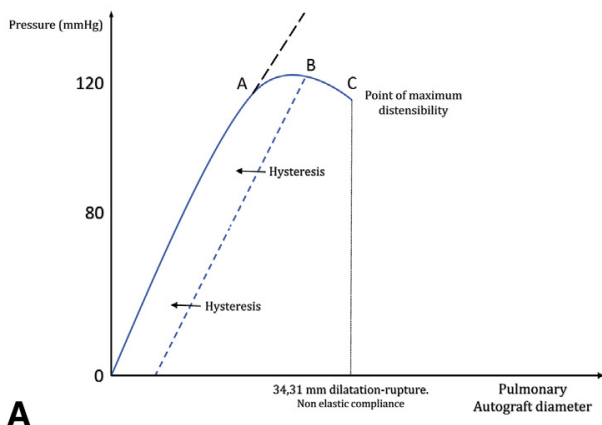

A

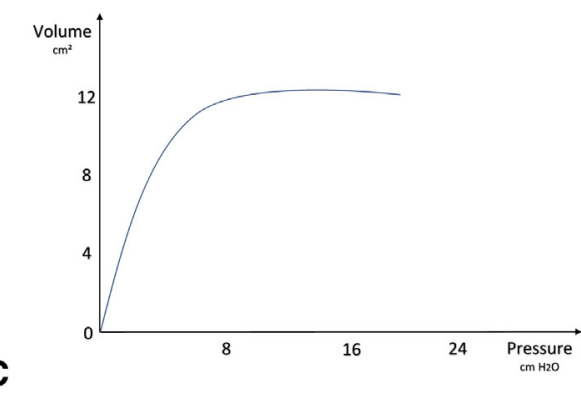

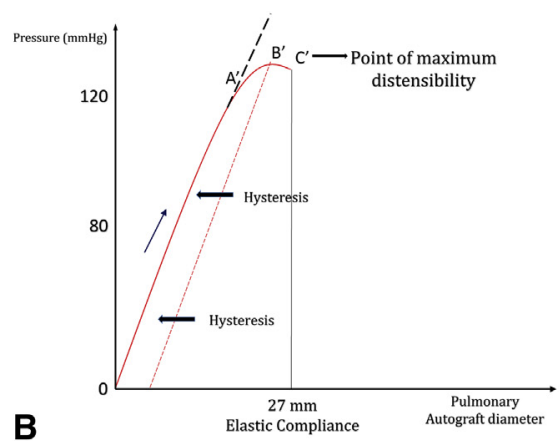

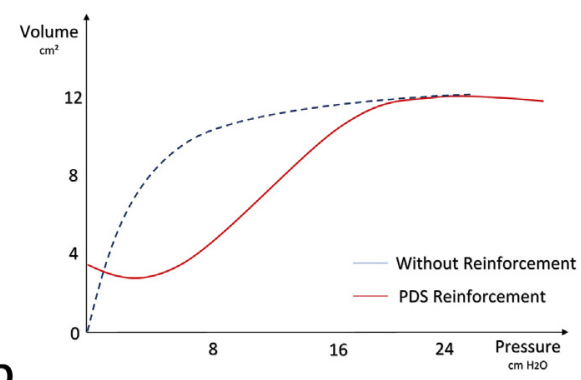

D

FIGURE 6. Top: Simple mathematic model and fitting curves based on the neo-Hookean hyperelastic behavior of the vessel, as reported in the text. Top left: A, Without reinforcement. In the graph. $\mathrm{A}=$ term of the elastic behavior; $\mathrm{B}=$ nonelastic behavior; $\mathrm{C}=$ maximum distensibility. Top right: $\mathrm{B}$, With resorbable reinforcement. PDS used as a constituent of a crosslinked bioresorbable external reinforcement can modify the behavior of the curve of distensible materials. Promoting the elastic remodeling, PDS influenced the elastic region of the graph promoting a shift of points A, B, and C, eventually reducing the critical area determined by the fall of the curve. Bottom: Elastic behavior and expected pressure-volume curves in the case of vessel deformation and growth; bottom left: $\mathrm{C}$, nonreinforced case; bottom right: $\mathrm{D}$, comparison between reinforced and nonreinforced autograft. PDS, Polydioxanone.

distensible membranes, as the PA conduit, in terms of elastic tension. The elastic tension for a solid is dependent on the surface and its intrinsic characteristic (ie, vessel wall properties). When the PA is transposed in the aortic position, the systemic pressure determines a conversion of the elastic tension in elastic potential energy. Therefore, the potential elastic energy depends on the extension of the surface and on the composition of the material constituting the cylinder including the intramolecular cohesion forces. We demonstrated histologically that during the resorption process of a PDS scaffold applied to the PA, a remodeling process of the vessel wall occurs, resulting in a more dense connective architecture of the tunica media with an increase in its elastic component. We can reliably speculate that this phenomenon fits in the mathematic model and that the bioresorbable prosthesis increased the surface area of the neoaortic membrane and, at the same time, being resorbed within the PA, modified both the forces of cohesion and the elastic characteristics of the membrane (Figure 6, C and $D$ ). The molecular structure of PDS is characterized by repetition of fundamental units with a crystallinity of $55 \%$ circa, being likely to increase the intramolecular cohesion forces. ${ }^{24}$

The autograft morphologic and geometric features, nonhomogeneous elastic characteristics, and time evolution of the mechanical properties and deformation processes expected during growth and remodeling of the tissue, in both reinforced and nonreinforced cases, contribute to complex dynamics. Nevertheless, some ad hoc simplified schemes, governed by suitable geometric and mechanical parameters, can be realized to determine key factors that mainly influence the biomechanical response of both reinforced and nonreinforced PAs under the action of the systemic pressure. In particular, by making reference to the overall mechanical properties and mean initial autograft diameter $d$ and wall thickness $t$ of an equivalent elastic cylindrical tube (eg, the comprehensive work on growth and elasticity of arterial walls by Holzapfel and Ogden ${ }^{25}$ ) under the hypothesis of neo-Hookean incompressible hyperelastic behavior and exploiting the Laplace formula, one can mathematically derive the following relationship between the internal pressure $p$ and the final deformed diameter $D$ through the circumferential stress $s$ :

$$
p=s \times \frac{2 T}{D} \Rightarrow p=G \times\left[1-\left(\frac{d}{D}\right)^{4}\right] \times \frac{2 t}{d}
$$

where $T$ is the final thickness and $G$ is the Lamè elastic shear modulus.

The aortic root anatomy presents an increased degree of complexity and cannot be approximated to a cylindric geometry. As described earlier, material deformation occurs 


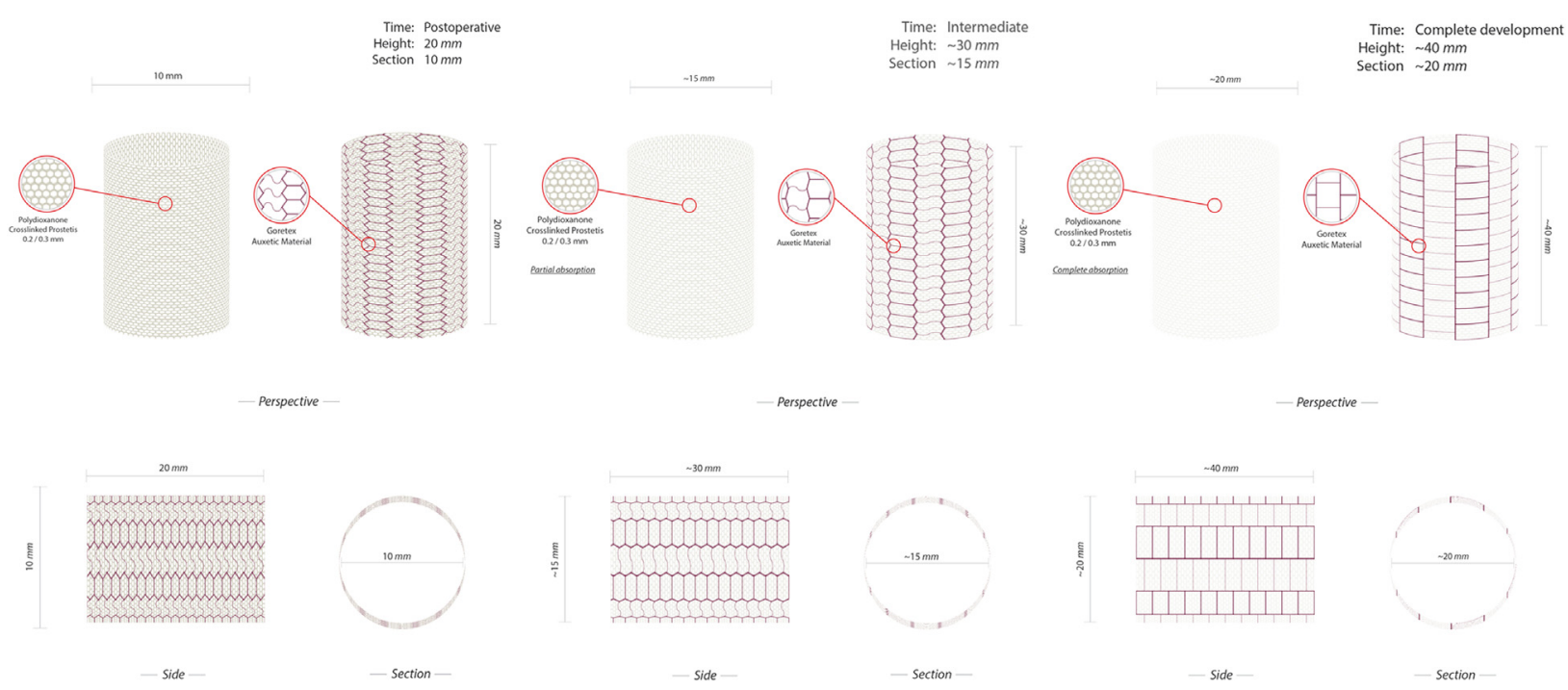

FIGURE 7. Concept and design of composite semiresorbable armored bioprosthesis. The specific design of the Gore-Tex armor will allow multidirectional growth and resistance to dilatation (see text). The particular weave of the superior part of the armor (left) will progressively adapt and functionally compensate the characteristics of autograft growth. Left: Initial implantation. Center: Intermediate phase. Right: complete development. Note progressive resorption of the resorbable layer and progressive expansion of unitary elements composing the mesh composed by the auxetic material ePTFE.

in axial and lateral fashions, but also a shear stress modulus is applied to determine a sliding of the conduit components. Clinical reports on the differential enlargement of the different sectors of the root and their potential for dilation ${ }^{15}$ made us reconsider the PA reinforcement strategy on the basis of the mathematic model developed and on our initial experience on resorbable reinforcement. The major disadvantages of synthetic materials used in pediatric cardiovascular surgery regard the inability to adapt to the vessels during their structural development and growth, and the induction of a strong inflammatory reaction that affects the viability of the autografts interfering with the normal process of arterialization and impairs their elastic compliance. $^{12,19}$ For these reasons, we needed to select a material suitable to comply with both shear modulus requirements and differential dilation tendency of the root. ePFTE is a material currently used in surgery and known, from the elastomechanical standpoint, to have a so-called auxetic behavior. Its Poisson's ratio, that is, the parameter to describe the ability of a material to respond to applied forces, is negative, which provides it with extremely advantageous compliance properties. ePTFE fibers, if subjected to a tensile stress, open up structurally and expand in the direction transverse to the stress; conversely, if these materials are subjected to compression, they close structurally. As shown in Figure 7, we designed a specific ePTFE external armour not in direct contact with the vessel wall, which would progressively allow multidirectional growth and resistance to dilatation over time. The plurality of interconnected transverse and longitudinal wires constitutes a mechanism to adapt to longitudinal growth and dilation going progressively from a "loose" to an "extended" configuration. Therefore, the specific weave design would be able to address issues of differential tendency to dilation of the different components of the aortic root. In this context, the resorbable material used, PDS, plays a key role in the architecture of the proposed prosthesis system. It works on 2 levels: At the early stage, its mechanical properties serve to add elastic stiffness to the trunk for confining exceeding vessel dilation phenomena and avoiding aneurysms; successively, during its degradation process, the PDS slowly abandons its initial stiffening role to allow pressure-induced PA tissue remodeling and growth that will finally lead to the vessel arterialization, the overall result depending on the synergy between the PDS and the ePTFE materials. From the biomechanical standpoint, it can be envisaged that a virtuous cooperation between biological and synthetic materials takes place, a sort of "stress-shielding" phenomenon guiding the physiologic arterialization of the vessel wall, which finally determines the success of the autograft system. In particular, the doubly reinforced PA prosthesis system would work as a sort of "relay race" by guaranteeing the handover of the bearing structure functions from the PDS scaffold (at the early stages of tissue growth and remodeling) to the ePTFE armor, initially "dormant" as a consequence of the typically low stiffness exhibited by auxetic structures at small strain. In this way, the mechanical shielding of the lapse vessel contains the radial expansion and simultaneously allows the pulmonary artery to remodel its tunics to attain an adequate level of mechanical properties. Once the bioresorbable scaffold has completed its degradation 
program and the strengthened vessel walls can actively respond to the systolic pressure, the ePTFE structure accompanies the PA media and adventitia toward their progressive aortic somatic growth by stretching its weave to gain stiffness and effectively confine further vessel expansion, thus avoiding tissue prolapse and aneurysmal degenerative phenomena.

At the end of the study (6 months), we obtained neoaortic diameters in the reinforced group comparable to those reported by Bonhoeffer and colleagues ${ }^{26}$ in the ascending aorta of juvenile lambs at the same age. This point might acquire additional relevance considering that in the Ross procedure the pulmonary trunk is not transposed in the descending aorta (as in this experimental model) but in the aortic root, and the ascending aorta diameter represents the most accurate reference point to be taken because it is the site of the actual anastomosis with the PA and more accurately reflects the clinical situation.

\section{CONCLUSIONS}

The device adapted and functionally compensated for the characteristics of autograft growth, guaranteeing a reasonable size of the autograft at 6 months, but more important, because the device is biocompatible, it did not disrupt the biological process of growth or cause inflammatory damage to the wall. The material harmoniously integrated with the vessel wall and induced an elastic remodeling, which is likely to have attenuated the pressure load exerted on the PA and compensated for the tendency to dilation, assisting somatic growth and preventing aneurysmal degeneration.

\section{Conflict of Interest Statement}

Authors have nothing to disclose with regard to commercial support.

The authors thank Dr Yves Lecompte for expert consultation. Without his advice, we could not have achieved these results. The authors also thank Matrix Consulting Ltd (Vittorio Paone) for graphical rendering.

\footnotetext{
References

1. Stelzer P. The Ross procedure: state of the art 2011. Semin Thorac Cardiovasc Surg. 2011;23:115-23.

2. David TE. Ross procedure at the crossroads. Circulation. 2009;119:207-9.

3. David TE, David C, Woo A, Manlhiot C. The Ross procedure: outcomes at 20 years. J Thorac Cardiovasc Surg. 2014;147:85-93.

4. Charitos EI, Takkenberg JJ, Hanke T, Gorski A, Botha C, Franke U, et al. Reoperations on the pulmonary autograft and pulmonary homograft after the Ross procedure: an update on the German Dutch Ross Registry. J Thorac Cardiovasc Surg. 2012;144:813-23.

5. Ross D. Pioneers of cardiology: Donald Ross, DSc, FRCS. Interview by Mark Nicholls. Circulation. 2007;115:f33-4.
}

6. Konstantinov IE. Is Ross operation in neonates and infants justified? Aortic valve repair may postpone Ross operation. Eur J Cardiothorac Surg. 2015;47: e170-1.

7. Hanke T, Stierle U, Boehm JO, Botha CA, Matthias Bechtel JF, Erasmi A, et al. Autograft regurgitation and aortic root dimensions after the Ross procedure: the German Ross Registry experience. Circulation. 2007;116:I251-8.

8. Charitos EI, Hanke T, Stierle U, Robinson DR, Bogers AJ, Hemmer W, et al. Autograft reinforcement to preserve autograft function after the Ross procedure: a report from the German-Dutch Ross registry. Circulation. 2009;120:S146-54.

9. Al Rashidi F, Bhat M, Hoglund P, Meurling C, Roijer A, Koul B. The modified Ross operation using a Dacron prosthetic vascular jacket does prevent pulmonary autograft dilatation at 4.5-year follow-up. Eur J Cardiothorac Surg. 2010;37: 928-33.

10. Koul B, Al-Rashidi F, Bhat M, Meurling C. A modified Ross operation to prevent pulmonary autograft dilatation. Eur J Cardiothorac Surg. 2007;31:127-8.

11. Gebauer R, Cerny S. A modification of the Ross procedure to prevent pulmonary autograft dilatation. Eur J Cardiothorac Surg. 2009;36:195-7.

12. Nappi F, Spadaccio C, Al-Attar N, Acar C. The Ross procedure at the crossroads: lessons from biology: is Dr Ross's dream concluded? Int J Cardiol. 2015;178: 37-9.

13. Nappi F, Spadaccio C, Castaldo C, Di Meglio F, Nurzynska D, Montagnani S, et al. Reinforcement of the pulmonary artery autograft with a polyglactin and polydioxanone mesh in the Ross operation: experimental study in growing lamb. $J$ Heart Valve Dis. 2014;23:145-8.

14. Nappi F, Spadaccio C, Fouret P, Hammoudi N, Chachques JC, Chello M, et al. An experimental model of the Ross operation: development of resorbable reinforcements for pulmonary autografts. J Thorac Cardiovasc Surg. 2015; 149:1134-42.

15. Horer J, Hanke T, Stierle U, Takkenberg JJ, Bogers AJ, Hemmer W, et al. Neoaortic root diameters and aortic regurgitation in children after the Ross operation. Ann Thorac Surg. 2009;88:594-600.

16. Spadaccio C, Rainer A, Barbato R, Chello M, Meyns B. The fate of largediameter Dacron $(\mathrm{R})$ vascular grafts in surgical practice: are we really satisfied? Int J Cardiol. 2013;168:5028-9.

17. Lu S, Zhang P, Sun X, Gong F, Yang S, Shen L, et al. Synthetic ePTFE grafts coated with an anti-CD133 antibody-functionalized heparin/collagen multilayer with rapid in vivo endothelialization properties. ACS Appl Mater Interfaces. 2013:5:7360-9.

18. Rechtsman MC, Stillinger FH, Torquato S. Negative Poisson's ratio materials via isotropic interactions. Phys Rev Lett. 2008;101:085501.

19. Carrel T, Schwerzmann M, Eckstein F, Aymard T, Kadner A. Preliminary results following reinforcement of the pulmonary autograft to prevent dilatation after the Ross procedure. J Thorac Cardiovasc Surg. 2008;136:472-5.

20. Carrel T. The autograft inclusion: an obligatory step to avoid late failure following the Ross procedure? J Thorac Cardiovasc Surg. 2015;149:S53-4.

21. Slater M, Shen I, Welke K, Komanapalli C, Ungerleider R. Modification to the Ross procedure to prevent autograft dilatation. Semin Thorac Cardiovasc Surg Pediatr Card Surg Annu. 2005;181-4.

22. Husain SA. The continued evolution of a transformational operation: new options for wrapping the Ross autograft? J Thorac Cardiovasc Surg. 2015;149:1142-3.

23. Spadaccio C, Montagnani S, Acar C, Nappi F. Introducing bioresorbable scaffolds into the show. A potential adjunct to resuscitate Ross procedure. Int J Cardiol. 2015;190:50-2.

24. Boland ED, Coleman BD, Barnes CP, Simpson DG, Wnek GE, Bowlin GL. Electrospinning polydioxanone for biomedical applications. Acta Biomater. 2005;1: 115-23.

25. Holzapfel GA, Ogden RW. Constitutive modelling of arteries. Proc Math Phys Eng Sci. 2010;466:1551-97.

26. Bonhoeffer P, Boudjemline Y, Saliba Z, Hausse AO, Aggoun Y, Bonnet D, et al. Transcatheter implantation of a bovine valve in pulmonary position: a lamb study. Circulation. 2000;102:813-6.

Key Words: Ross operation, pulmonary autograft, tissue engineering, stress shielding, aortic remodeling 


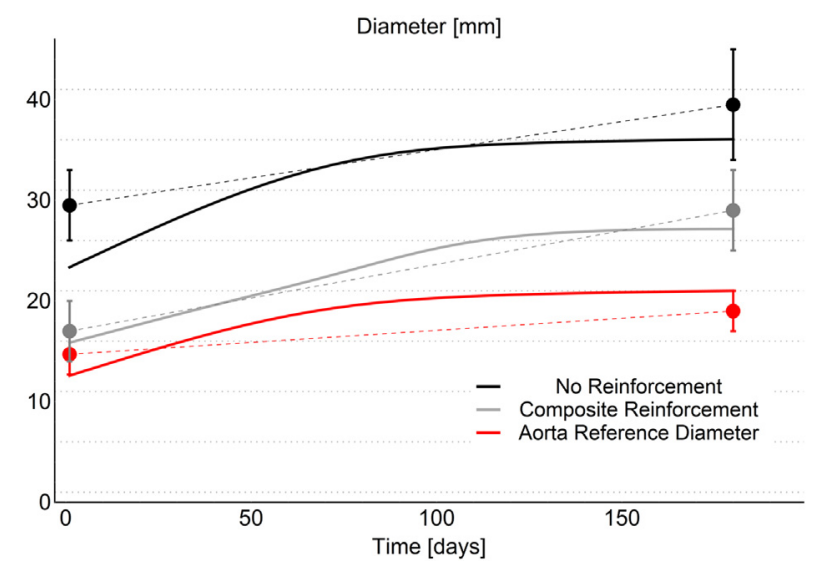

FIGURE E1. Graph showing the evolution of the outer diameters (continuous lines represent theoretical outcomes while markers are experimental measures). In a parallel biomechanical study, a nonlinear Gompertzian-like growth profile of the reinforced graft diameter and walls have been found, showing that the velocity of the process at hand varies with time and, in the case of reinforced pulmonary autograft, the sizes asymptotically tend to stabilize around physiologic dimensions of the control. The mathematical model describing this behavior has been elaborated and explained elsewhere. (Data from Nappi F, Carotenuto A, Di Vito D, Spadaccio C, Fraldi M. Stress-shielding and remodeling of pulmonary artery reinforced with scaffold copolymer and transposed into aortic position. Biomech Modeling Mechanobiol. 2015 [In press].) 